\title{
Effect of Net Charge on the Relative Stability of 2D Boron Allotropes
}

\author{
Dan Liu and David Tománek* \\ Physics and Astronomy Department, Michigan State University, East Lansing, Michigan \\ 48824, USA \\ E-mail: tomanek@pa.msu.edu
}

\section{Abstract}

We study the effect of electron doping on the bonding character and stability of twodimensional (2D) structures of elemental boron, called borophene, which is known to form many stable allotropes. Our ab initio calculations for the neutral system reveal previously unknown stable $2 \mathrm{D} \epsilon-\mathrm{B}$ and $\omega$-B structures. We find that the chemical bonding characteristic in this and other boron structures is strongly affected by extra charge. Beyond a critical degree of electron doping, the most stable allotrope changes from $\epsilon$-B to a buckled honeycomb structure. Additional electron doping, mimicking a transformation of boron to carbon, causes a gradual decrease in the degree of buckling of the honeycomb lattice that can be interpreted as piezoelectric response. Net electron doping can be achieved by placing borophene in direct contact with layered electrides such as $\mathrm{Ca}_{2} \mathrm{~N}$. We find that electron doping can be doubled by changing from the $\mathrm{B} / \mathrm{Ca}_{2} \mathrm{~N}$ bilayer to the $\mathrm{Ca}_{2} \mathrm{~N} / \mathrm{B} / \mathrm{Ca}_{2} \mathrm{~N}$ sandwich geometry.

\section{Keywords}

ab initio calculation, electronic structure, doping, boron, structural stability, 2D

Identifying the most stable allotrope of a given compound is one of the key problems in Physics and Chemistry. Whereas charge neutral systems have attracted most atten- tion, notable exceptions are low-dimensional systems that can be charged by doping in specific bulk geometries. Electron doping induced by Li intercalation does not affect the honeycomb structure of two-dimensional (2D) graphene layers in graphite intercalation compounds (GICs), 11 but does change the equilibrium structure of $\mathrm{MoS}_{2}$ monolayers from the $2 \mathrm{H}$ to the $1 \mathrm{~T}$ allotrope. ${ }^{2}$ Even though the effect of excess charge on chemical bonding and equilibrium geometry should be general, we expect the most drastic changes to occur in structures of elemental boron that is notorious for its many stable allotropes. ${ }^{3 / 4}$ What appears as frustrated bonding in mostly metallic 2D structures of elemental boron, called borophene, reflects the inability of the element to follow the octet rule of bonding due to its electron deficiency. We speculate that both the electron deficiency and thus the chemical bonding characteristic may be modified by placing a nonzero charge on $\mathrm{B}$ atoms. In that case, the most stable borophene structure may differ from a puckered triangular lattice with monatomic vacancies ${ }^{5-9}$ or equally stable irregular structures ${ }^{10 \mid 12}$ identified in the neutral system. Indeed, placing a net charge of $\lesssim 1$ e per atom on borophene, provided by an $\mathrm{Al}(111)$ substrate, ${ }^{13}$ or intercalating $\mathrm{Mg}$ ions in-between borophene layers in the $\mathrm{MgB}_{2}$ compound,, 14 changes the most stable allotrope to a very different honeycomb lattice. Structural changes induced by charging may significantly modify the electronic structure, turning semiconducting $2 \mathrm{H}-\mathrm{MoS}_{2}$ to metallic $1 \mathrm{~T}$ '- $-\mathrm{MoS}_{2}$ lo- 
cally 15 and undoped borophene to honeycomb lattices in diborides ${ }^{16 / 17}$ including $\mathrm{MgB}_{2}$, which displays superconducting behavior. $\frac{18}{}$

In this study, we explore the effect of net charge on the bonding character and structural stability of 2D allotropes of boron. Our $a b i n i-$ tio calculations for the neutral system reveal a previously unknown stable $2 \mathrm{D} \epsilon$-B structure with a $0.2 \mathrm{eV}$ wide fundamental band gap. We find that the chemical bonding characteristic in this and other boron structures is strongly affected by extra charge, including a $23 \%$ lattice constant change in $\epsilon$-B, induced by changing the net charge from 0.25 holes to 0.25 electrons per B atom. Beyond a critical degree of doping near 0.5 electrons/atom, the most stable allotrope changes from $\epsilon$-B to a buckled honeycomb structure. Additional electron doping, mimicking a transformation of boron to carbon, causes a gradual decrease in the degree of buckling of the honeycomb lattice that can be interpreted as piezoelectric response. We propose that net electron doping can easily be achieved by placing borophene in direct contact with layered electrides such as $\mathrm{Ca}_{2} \mathrm{~N}$. In this system, we find that electron doping of borophene can be doubled by changing from the $\mathrm{B} / \mathrm{Ca}_{2} \mathrm{~N}$ bilayer to the $\mathrm{Ca}_{2} \mathrm{~N} / \mathrm{B} / \mathrm{Ca}_{2} \mathrm{~N}$ sandwich geometry.

As mentioned above, the vast number of stable neutral 2D borophene allotropes, including $\alpha-\mathrm{B}, \beta-\mathrm{B}, \gamma-\mathrm{B}, \delta-\mathrm{B}$ and $\eta-\mathrm{B}$ structures, ${ }_{516}^{5}$ reflects a frustrated bonding character of electron-deficient boron. This element may engage its three valence electrons in only three covalent bonds, resulting in an electron sextet instead of the desirable octet noble-gas configuration. To partly compensate for lack of electrons in the octet configuration, boron atoms often prefer increasing their coordination to six nearest neighbors in the triangular lattice. This is essentially equivalent to adding electrons ${ }^{19 / 20}$ to the individual atoms that are held together by pure three-center bonding. On the other hand, the stability of the triangular lattice is often enhanced by removing atoms and forming hexagon-shaped monatomic vacancies. This process somehow mimics subtracting electrons while the structure locally converts to a honeycomb lattice, where atoms are held together by two-center bonding. The competition between two-center and three-center bonding has been used to identify the optimum concentration of hexagonal vacancies in the neutral triangular

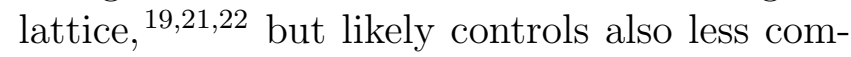
mon neutral structures with four- and five-fold coordinated boron atoms. $\frac{10111}{1}$ Biasing this competition by net charge has been shown to affect the fraction of hexagonal vacancies in triangular borophene lattices. ${ }^{23}$ Even though doping of B layers has been linked to unexpected superconducting behavior of $\mathrm{MgB}_{2}$ and found useful to modulate $\mathrm{CO}_{2}$ capture, ${ }^{24}$ no systematic attention has been paid to the possibility of deliberately changing the bonding and the equilibrium structure of boron by excess charge.

A viable possibility to significantly dope $2 \mathrm{D}$ structures of elemental boron by electrons is to place them in direct contact with electrides including $\mathrm{Ca}_{2} \mathrm{~N}^{25 \mid 26}$ and $\mathrm{Y}_{2} \mathrm{C}$. 27 In these highly electronegative systems with a layered structure, regions of large electron density are found in-between the layers. The charge density in this electron layer amounts to one electron per formula unit in $\mathrm{Ca}_{2} \mathrm{~N}$ and two electrons per formula unit in $\mathrm{Y}_{2} \mathrm{C}$. The possibility of exfoliation down to a monolayer ${ }^{[25}$ has been demonstrated in $\mathrm{Ca}_{2} \mathrm{~N}$, so that assembly of various vertical heterostructures is possible.

\section{Results}

\section{Allotropes of neutral and doped borophene}

Inspired by the honeycomb structure of the negatively doped boron sublattice found in diborides including $\mathrm{MgB}_{2}$, we started our investigation of electron doped borophene structures with the honeycomb lattice. To provide substantial configuration freedom for the lattice structure, we consider a superlattice with 32 atoms per unit cell and subject all atoms in the supercell to random distortion. To study the effect of doping on the equilibrium structure and the chemical nature of bonding, we changed the degree of doping gradually and optimized each system using the conjugate gradient optimiza- 


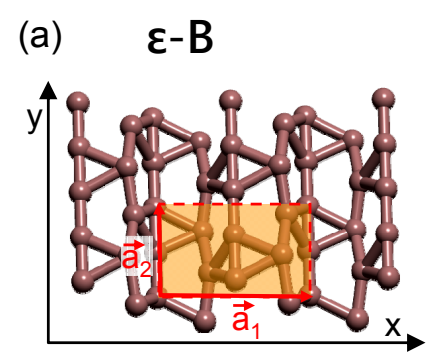

(b)
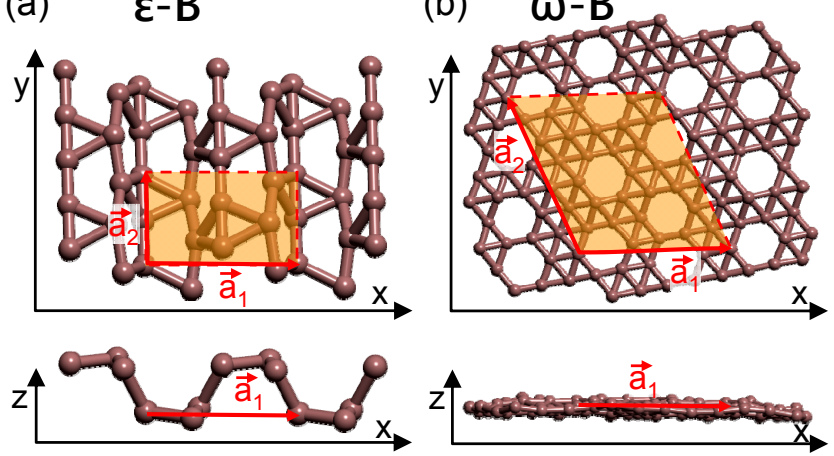

Figure 1: Previously unexplored neutral borophene allotropes formed by spontaneous conversion of an artificial honeycomb lattice: (a) $\epsilon$-B and (b) $\omega$-B. The structures are shown in top and side view. The lattice vectors $\vec{a}_{1}$ and $\vec{a}_{2}$, shown in red, delimit the highlighted primitive unit cells.

tion method.

Starting with no excess charge, we found the neutral honeycomb structure to be unstable and to convert to rather stable allotropes that have not been reported previously. The first allotrope, shown in Fig. 1(a) and called $\epsilon$-B, is only $7 \mathrm{meV} /$ atom less stable than the most stable $\alpha^{\prime}-\mathrm{B}$ phase $\mathrm{e}^{3}$ and displays a very uncommon morphology with triangles and pentagons,

Table 1: Cohesive energy $E_{c o h}$ of selected neutral borophene allotropes, obtained using DFTPBE calculations. $n$ is the number of boron atoms per unit cell and $Z$ is the coordination number of individual atoms in the unit cell.

\begin{tabular}{lccc}
\hline \hline Allotrope & $E_{\text {coh }}(\mathrm{eV} /$ atom $)$ & $n$ & $Z$ \\
\hline$\epsilon$-B & $5.699^{a}$ & 8 & 4 \\
$\omega-\mathrm{B}$ & $5.680^{a}$ & 32 & $4,5,6$ \\
$\alpha^{\prime}-\mathrm{B}$ & $5.706^{a}$ & 8 & 5,6 \\
& $5.762^{b}$ & 8 & 5,6 \\
$\beta_{12}-\mathrm{B}$ & $5.712^{b}$ & 5 & $3,5,6$ \\
& $6.23^{c}$ & 5 & $3,5,6$ \\
$\delta_{6}-\mathrm{B}$ & $5.662^{b}$ & 4 & 6 \\
$\chi_{3}-\mathrm{B}$ & $5.723^{b}$ & 4 & 3,5 \\
& $6.19^{c}$ & 4 & 3,5 \\
\hline \hline
\end{tabular}

\footnotetext{
${ }^{a}$ Present work.

${ }^{b}$ Reference [3].

${ }^{c}$ Reference [8].
}

(a) $\angle \Delta Q>=-0.156 \mathrm{e} / \mathrm{B}$-atom

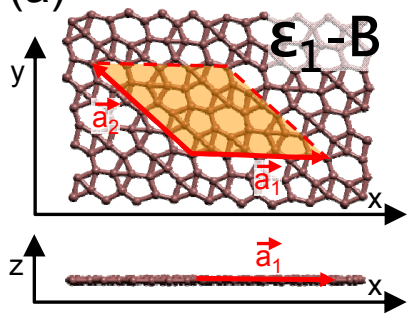

(b) $\langle\Delta Q\rangle=-0.312$ e/B-atom

(c) $\langle\Delta Q\rangle=-0.406$ e/B-atom



(e) $\langle\Delta Q>=-0.750$ e/B-atom
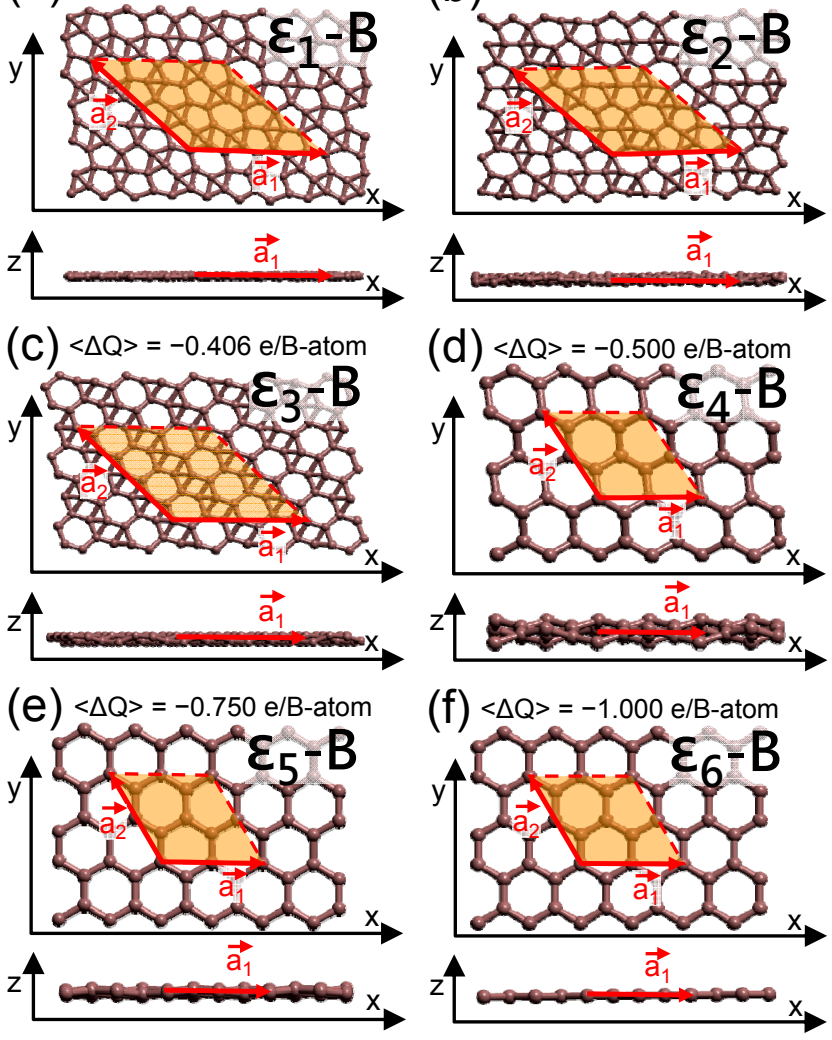

(d) $\langle\Delta Q\rangle=-0.500$ e/B-atom



(f) $\langle\Delta Q>=-1.000$ e/B-atom

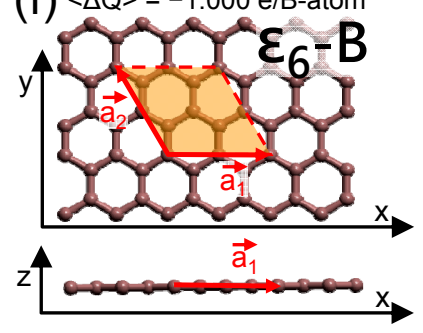

Figure 2: Electron doped 2D borophene structures obtained by optimizing a distorted boron honeycomb superlattice with 32 atoms per unit cell. The average excess charge $\langle\Delta Q\rangle$ per boron atom, specified in the panels, increases from (a) $\epsilon_{1}$-B to (f) $\epsilon_{6}$-B. The structures are shown in top and side view. The lattice vectors $\vec{a}_{1}$ and $\vec{a}_{2}$, shown in red, delimit the highlighted unit cells.

quite distinct from the well-documented class of triangular lattices with vacancies. The structure shown in Fig. 1(b), which we call $\omega-\mathrm{B}$, has a very similar morphology to known allotropes containing triangles and hexagons only, 54 but is $26 \mathrm{meV} /$ atom less stable than $\alpha^{\prime}-\mathrm{B}$. $\epsilon-\mathrm{B}$ and $\omega$-B add to the large number of known allotropes, and we expect many more to follow.

Both $\epsilon$-B and $\omega$-B are buckled. Still, we could locate a locally stable, flat counterpart of $\omega$ $\mathrm{B}$, which is $7 \mathrm{meV}$ /atom less stable than $\omega$ $\mathrm{B}$. The cohesive energies of neutral borophene allotropes are compared in Table 1 .

Next, we added extra 5, 10,13, 16, 24 and 32 electrons to the 32-atom unit cell with an initial honeycomb arrangements and optimized 
the geometry. We found the optimum geometries not to depend on initial deformations imposed on the starting structure, which may not be reachable in molecular dynamics trajectories. The optimum boron structures labelled $\epsilon_{1}-\epsilon_{6}$ and their average doping levels $\langle\Delta Q\rangle$ are displayed in Figs. 2(a)-2(f). Optimum structures for different doping levels are discussed in the Supporting Information (SI). We find all these structures to differ significantly from the neutral $\epsilon$-B and $\omega$-B structures in Fig. 1, which have been optimized in the same way.

The structure $\epsilon_{1}-\mathrm{B}$ in Fig. 2(a), which contains triangles, pentagons and hexagons, is reminiscent of $\epsilon$-B, but is completely flat. With increasing electron doping, the density of pentagons gradually diminishes and eventually vanishes in $\epsilon_{3}$-B in Fig. 2(c), representing the buckled $\chi_{3}$ phase. ${ }^{3}$ At the same time, the density of hexagons increases from $\epsilon_{1}$-B to $\epsilon_{3}$-B until all other polygons are eliminated in $\epsilon_{4}-\epsilon_{6}$, shown in Figs. 2(d)-2(f), as the doping level exceeds $|\langle\Delta Q\rangle| \gtrsim 0.5 \mathrm{e}$ /atom. The buckled honeycomb lattice of $\epsilon_{4}$-B gradually flattens to the graphene-like structure of $\epsilon_{6}$-B with increasing electron doping.

The interpretation of these structural changes is rather straight-forward. With one extra electron per atom, boron behaves as $s p^{2}$-bonded carbon with four valence electrons, with atoms forming the 2D graphene honeycomb lattice. This structure is rather robust with respect to electron and hole doping, as evidenced in GIC structures. Similarly, also doped boron should keep its optimum honeycomb lattice structure even if the net charge may be smaller or larger than one extra electron per atom. As seen in Figs. 2(d)-2(f), the honeycomb structure of borophene, with different degree of buckling, is preferred for the excess charge $\langle\Delta Q\rangle$ ranging between -0.5 and -1.0 e/atom.

To understand the change in the electronic structure that caused a profound structural change from neutral $\epsilon$-B in Fig. 1(a) to negatively charged $\epsilon_{6}-\mathrm{B}^{-}$in Fig. 2(f), we first probed the charge density changes $\Delta \rho$ associated with charging. Our results for $\Delta \rho$ caused by placing artificially extra electrons on free-standing borophene structures $\epsilon_{3}$-B and $\epsilon_{6}$-B are shown in Figs. 3(a)-3(b). $\Delta \rho$ can be viewed as the crystal counterpart of the Fukui function, and our results indicate that the extra electrons are accommodated in $p_{z}$ (or $\pi$ ) states normal to the layer, very similar to graphene layers.

A realistic way to provide a high degree of electron doping, we suggest, is to place borophene layers in contact with the $\mathrm{Ca}_{2} \mathrm{~N}$ electride. The optimum lattice constant of the triangular lattice of $\mathrm{Ca}_{2} \mathrm{~N}$ is $a=3.97 \AA$. This layered system has the nominal configuration $\left[\mathrm{Ca}_{2} \mathrm{~N}\right]^{+} \cdot \mathrm{e}^{-}$, with layers of $\mathrm{Ca}_{2} \mathrm{~N}$ separated by layers of excess electrons, and can be exfoliated down to a monolayer chemically. $\frac{25}{2 t}$ is to be expected that borophene will be electron doped when placed in the region of excess electrons outside a $\mathrm{Ca}_{2} \mathrm{~N}$ monolayer. Since we focus on general trends rather than minute details, we studied the charge redistribution using only two prototype structures of doped borophene, namely $\epsilon_{3}$-B at low- and $\epsilon_{6}$-B at high-level doping, in contact with $\mathrm{Ca}_{2} \mathrm{~N}$. To determine the degree of electron doping in borophene caused by a contact to $\mathrm{Ca}_{2} \mathrm{~N}$, we inspected the charge redistribution when placing $\epsilon_{3}-\mathrm{B}$ and $\epsilon_{6}$-B on top of a $\mathrm{Ca}_{2} \mathrm{~N}$ monolayer or, as in a sandwich geometry, in-between $\mathrm{Ca}_{2} \mathrm{~N}$ monolayers. The charge density differences caused by electron redistribution in the system are shown in Figs. 3(c)$3(\mathrm{f})$. Additional results for $\epsilon_{3}-\mathrm{B}$ and $\epsilon_{6}-\mathrm{B}$ on top of a $\left[\mathrm{Ca}_{2} \mathrm{~N}\right]_{2}$ bilayer are presented in the SI.

We should point out that incommensurate vertical heterostructures formed of doped borophene and $\mathrm{Ca}_{2} \mathrm{~N}$ layers can not be represented accurately in a periodic structure used in our computational approach. In our calculation, we used the optimum interlayer distance $d=4.0 \AA$ in agreement with our results in Figs. 4(a) and 4(b). We furthermore matched $1 \times 3 \epsilon_{3}-\mathrm{B}$ supercells with $3 \times 7 \quad \mathrm{Ca}_{2} \mathrm{~N}$ supercells in the $\epsilon_{3}-\mathrm{B} / \mathrm{Ca}_{2} \mathrm{~N}$ superstructure and primitive unit cells of $\epsilon_{6}$-B with $3 \times 3$ supercells of $\mathrm{Ca}_{2} \mathrm{~N}$ in the $\epsilon_{6}-\mathrm{B} / \mathrm{Ca}_{2} \mathrm{~N}$ superstructure. The remaining lattice mismatch of $\lesssim 2 \%$ was accommodated by averaging the lattice constants of borophene and $\mathrm{Ca}_{2} \mathrm{~N}$. Due to this minor lattice distortion, results presented in Figs. 3(c)-3(f) may differ to a small degree from the charge redistribution in an incommensurate structure. Comparing 
(a)

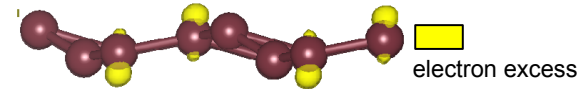

(c)
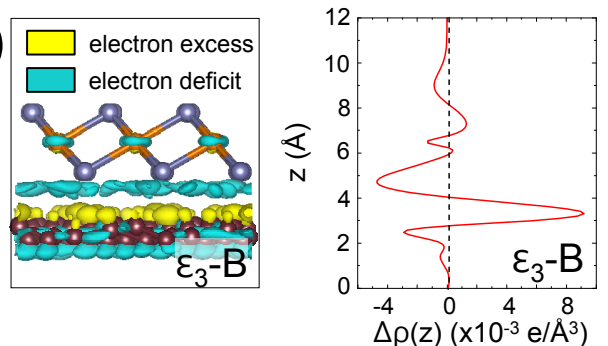

(d)



(b)
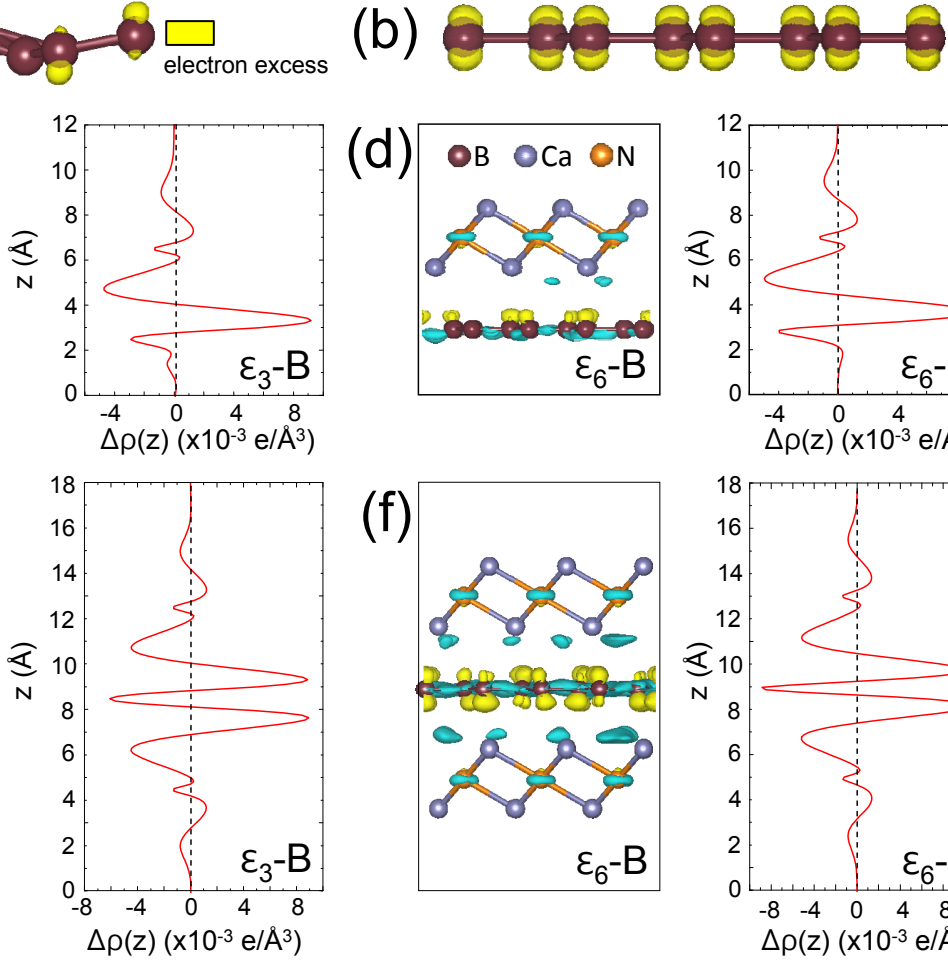

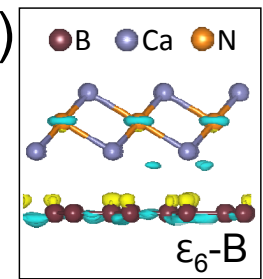

$\varepsilon_{6}-B$

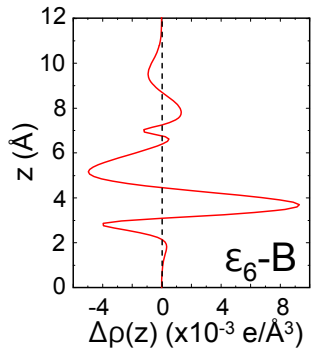

(f)



Figure 3: Charge redistribution in 2D borophene layers induced by electron doping or by contact with monolayers of $\mathrm{Ca}_{2} \mathrm{~N}$ electride. Charge density difference $\Delta \rho$ caused by placing an excess charge (a) $\langle\Delta Q\rangle=-0.406 \mathrm{e} /$ atom on $\epsilon_{3}-\mathrm{B}$ and $(\mathrm{b})\langle\Delta Q\rangle=-1.0 \mathrm{e} /$ atom on $\epsilon_{6}$ - $\mathrm{B}$. Charge density redistribution $\Delta \rho=\rho\left(\mathrm{B} / \mathrm{Ca}_{2} \mathrm{~N}\right)-\rho(\mathrm{B})-\sum \rho\left(\mathrm{Ca}_{2} \mathrm{~N}\right)$ in the bilayer structures $(\mathrm{c}) \epsilon_{3}-\mathrm{B} / \mathrm{Ca}_{2} \mathrm{~N}$ and $(\mathrm{d})$ $\epsilon_{6}-\mathrm{B} / \mathrm{Ca}_{2} \mathrm{~N}$ as well as in the sandwich structures (e) $\mathrm{Ca}_{2} \mathrm{~N} / \epsilon_{3}-\mathrm{B} / \mathrm{Ca}_{2} \mathrm{~N}$ and (f) $\mathrm{Ca}_{2} \mathrm{~N} / \epsilon_{6}-\mathrm{B} / \mathrm{Ca}_{2} \mathrm{~N}$. $\Delta \rho$ is shown by isosurfaces bounding regions of electron excess at $+7 \times 10^{-3} \mathrm{e} / \AA^{3}$ (yellow) and electron deficiency at $-2 \times 10^{-3} \mathrm{e} / \AA^{3}$ (blue). $\langle\Delta \rho(z)\rangle$ is averaged across the $x-y$ plane of the layers.

results for the bilayer in Figs. $3(\mathrm{c})-3(\mathrm{~d})$ with those in the sandwich structure in Figs. 3(e)$3(f)$, we see clearly that borophene receives twice the number of electrons in the sandwich in comparison to the bilayer structure. Specifically, $\langle\Delta Q\rangle$ in $\epsilon_{3}$-B almost doubles from $-0.16 \mathrm{e} /$ atom in Fig. 3(c) to $-0.31 \mathrm{e} /$ atom in Fig. 3(e). Similarly, $\langle\Delta Q\rangle$ in $\epsilon_{6}$-B almost doubles from -0.21 e/atom in Fig. 3(d) to -0.41 e/atom in Fig. 3(f).

We have evaluated the dependence of the total energy on the interlayer distance $d$ in the $\mathrm{Ca}_{2} \mathrm{~N} / \mathrm{B} / \mathrm{Ca}_{2} \mathrm{~N}$ sandwich structure for the $\epsilon_{3}-\mathrm{B}$ and $\epsilon_{6}$-B allotropes and present our results in Figs. 4(a) and 4(b). In both structures, the optimum interlayer distance $d \approx 4.0 \AA$.

There is a significant electron accumulation with a maximum at $d \approx 3 \AA$ outside a freestanding $\mathrm{Ca}_{2} \mathrm{~N}$ electride layer, ${ }^{25}$ which is accommodated by an adjacent borophene layer.
In this case, the net charge on the borophene layer may be changed by changing the interlayer distance $d$. Our results for $\langle\Delta Q\rangle$ as a function of $d$ are presented in Fig. 4(c) for $\epsilon_{3}-\mathrm{B}$ and in Fig. 4(d) for $\epsilon_{6}$-B sandwiched in-between two $\mathrm{Ca}_{2} \mathrm{~N}$ layers. We find it interesting that $\langle\Delta Q\rangle$ decreases almost linearly with increasing interlayer distance.

At the equilibrium interlayer distance, we find $\langle\Delta Q\rangle \approx-0.31 \mathrm{e} / \mathrm{B}$-atom in the system with $\epsilon_{3}$ - $\mathrm{B}$ and $\langle\Delta Q\rangle \approx-0.41 \mathrm{e} / \mathrm{B}$-atom in the system with $\epsilon_{6}$-B. The higher value of $\langle\Delta Q\rangle$ in $\epsilon_{6}$ - $\mathrm{B}$ is associated with the better ability of this structure to accept electrons.

It is well known that in GICs, the net charge transferred from dopant atoms to the graphene layers changes the lattice constant. This change is relatively small, amounting to ${ }^{28} \Delta a / a \approx 0.8 \%$ in $\mathrm{KC}_{8}$ with $\langle\Delta Q\rangle \approx-0.125 \mathrm{e} / \mathrm{C}$-atom. Our corresponding results for the effect of doping on 



Figure 4: Changes in the interlayer interaction energy $\Delta E$ and net average charge $\langle\Delta Q\rangle$ on borophene in the $\mathrm{Ca}_{2} \mathrm{~N} / \mathrm{B} / \mathrm{Ca}_{2} \mathrm{~N}$ sandwich geometry as a function of the interlayer distance $d$. Results for $\epsilon_{3}$-B in (a) and (c) are compared to those for $\epsilon_{6}$-B in (b) and (d).

the lattice constants in the stable $\epsilon$-B allotrope are shown in Fig. 5(a). The lattice changes are anisotropic and larger than found in graphene. We find that electron doping expands the lattice more along the softer $\vec{a}_{1}$ direction than along the harder $\vec{a}_{2}$ direction. At the electron doping levels $\langle\Delta Q\rangle \approx-0.3$ to -0.5 e/B-atom discussed above for the $\mathrm{Ca}_{2} \mathrm{~N} / \mathrm{B} / \mathrm{Ca}_{2} \mathrm{~N}$ heterostructures, the lattice expansion exceeds $10 \%$.

The in-layer stiffness of neutral, electronand hole-doped $\epsilon$-B is addressed in Fig. 5(b). We used the shorthand notation 0 for the neutral system, - for $\langle\Delta Q\rangle=-0.125 \mathrm{e} / \mathrm{B}$ and + for $\langle\Delta Q\rangle=+0.125$ e/B doping. Irrespective of doping, the energy change due to in-layer strain $\sigma$ is much larger along the harder $\vec{a}_{2}$ direction than along the softer $\vec{a}_{1}$ direction. In terms of the $2 \mathrm{D}$ elastic constants, ${ }^{29]}$ we find for the softer $\vec{a}_{1}$ direction $c_{11}(-)=67.99 \mathrm{~N} / \mathrm{m}, c_{11}(0)=18.70 \mathrm{~N} / \mathrm{m}$, and $c_{11}(+)=29.98 \mathrm{~N} / \mathrm{m}$. Along the harder $\vec{a}_{2}$ direction we find $c_{22}(-)=446.79 \mathrm{~N} / \mathrm{m}, c_{22}(0)=$ $360.52 \mathrm{~N} / \mathrm{m}$, and $c_{22}(+)=338.58 \mathrm{~N} / \mathrm{m}$.
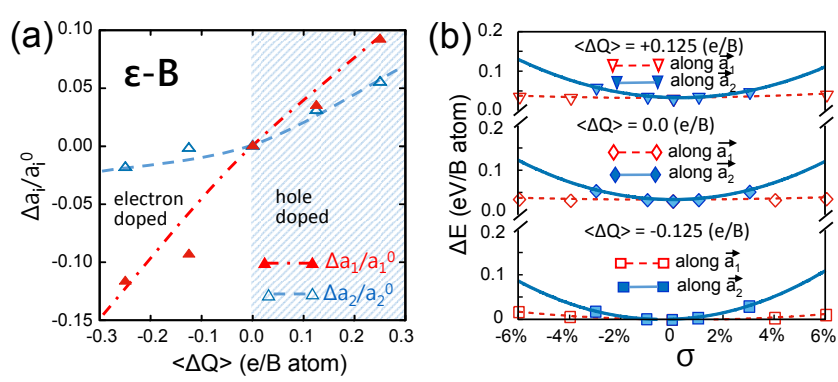

Figure 5: Effect of doping level on the equilibrium geometry of $\epsilon$-B. (a) Effect of the net average charge $\langle\Delta Q\rangle$ on the orthogonal lattice constants $a_{i}$ with $i=1,2$. Plotted are charge-induced relative changes $\Delta a_{i} / a_{i}^{0}$, where $a_{i}^{0}$ are the lattice constants in the neutral $\epsilon$-B allotrope. (b) Strain energy $\Delta E$ as a function of in-layer strain $\sigma$ at different doping levels $\langle\Delta Q\rangle$.

\section{Electronic structure of borophene allotropes}

The electronic band structure of selected boron 2D allotropes discussed in this study is shown in Fig. 6. We should note that DFT calculations used in this study do not represent the true quasi-particle band structure and typically underestimate band gaps. With this fact in mind, we find that, according to the PBE results in Fig. 6(a), the neutral $\epsilon$-B allotrope of Fig. 1(a) is a semiconductor with a small indirect band gap of $E_{g}=0.2 \mathrm{eV}$. Whereas stretching along the soft $\vec{a}_{1}$ direction by $4 \%$ turns $\epsilon$ $\mathrm{B}$ into a direct-gap semiconductor, compressing by $4 \%$ along $\vec{a}_{1}$ causes gap closure. Stretching $\epsilon$-B by $1 \%$ along the hard $\vec{a}_{2}$ direction turns this allotrope metallic, whereas compression by $1 \%$ changes the its indirect gap to a direct gap. More details about the electronic structure of strained $\epsilon$-B are provided in the SI.

We find all doped borophene allotropes to be metallic or semi-metallic, as shown in Figs. 6(b)-6(f). Inspection of the band structure reveals the formation of a Dirac cone at $\mathrm{K}_{1}$ and $K_{2}$ in the honeycomb structures in $\epsilon_{5}-\mathrm{B}$ in Fig. 6(e) and in $\epsilon_{6}$-B in Fig. 6(f). The Dirac cone appears $\approx 1.4 \mathrm{eV}$ above $E_{F}$ in $\epsilon_{5}-\mathrm{B}$ at the doping level $\langle\Delta Q\rangle=-0.750 \mathrm{e} / \mathrm{B}$ and at $E_{F}$ in $\epsilon_{6}-\mathrm{B}$ at $\langle\Delta Q\rangle=-1.000 \mathrm{e} / \mathrm{B}$, which mimics the structure and valence charge of graphitic car- 


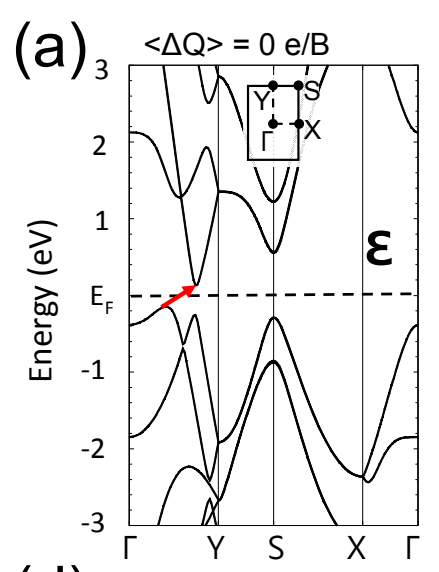

(d) $3<Q>=-0.500 \mathrm{e} / \mathrm{B}$

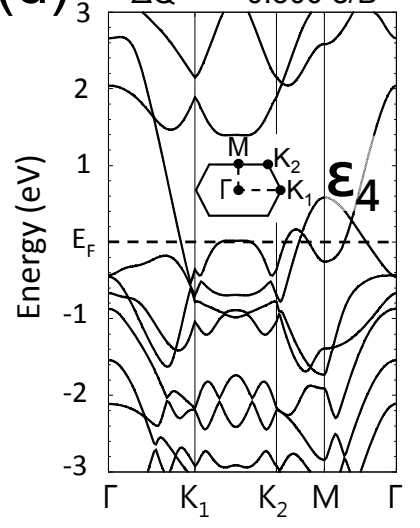

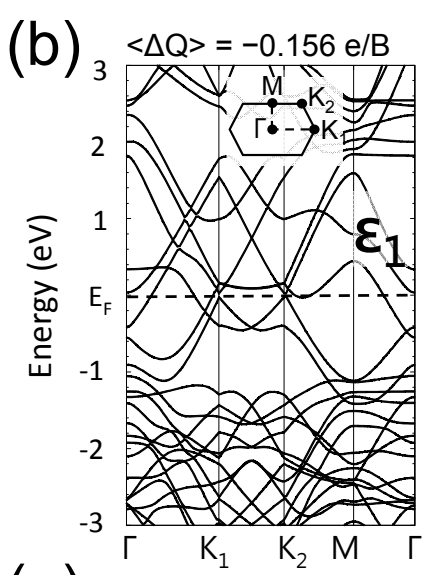

(e) $3<\Delta \mathrm{Q}>-0.750 \mathrm{e} / \mathrm{B}$



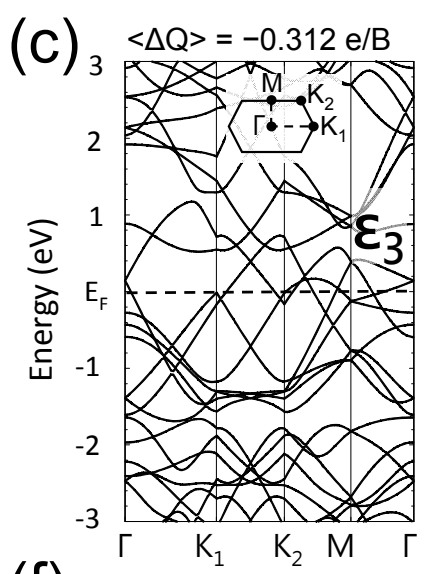

(f)

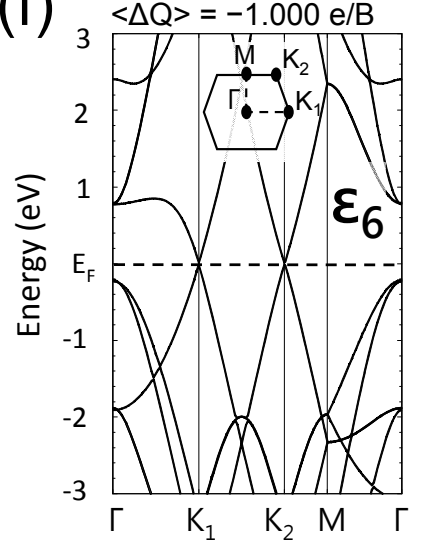

Figure 6: Electronic band structure of (a) neutral $\epsilon$-B, (b) $\epsilon_{1}$-B with $\langle\Delta Q\rangle=-0.156$ e/B, (c) $\epsilon_{3}$-B with $\langle\Delta Q\rangle=-0.406 \mathrm{e} / \mathrm{B}$, (d) $\epsilon_{4}-\mathrm{B}$ with $\langle\Delta Q\rangle=-0.500 \mathrm{e} / \mathrm{B}$, (e) $\epsilon_{5}-\mathrm{B}$ with $\langle\Delta Q\rangle=-0.750 \mathrm{e} / \mathrm{B}$, and (f) $\epsilon_{6}$-B with $\langle\Delta Q\rangle=-1.000 \mathrm{e} / \mathrm{B}$, calculated using the DFT-PBE functional. The indirect fundamental band gap is indicated by the red arrow in (a).

bon.

\section{Discussion}

The majority of the reported stable structures of neutral $2 \mathrm{D}$ borophene were triangular lattices containing arrays of monatomic vacancies, reflecting the frustrated bonding character of electron-deficient boron. When considering the effect of excess charge on the bonding geometry, we observed a transition driven by increasing net negative charge from structures containing triangles and higher polygons, depicted in Fig. 1(a) and Figs. 2(a)-2(c), for $|\langle\Delta Q\rangle|<0.5$ e/B to all-hexagon structures, depicted in Figs. 2(c)-2(f), for $|\langle\Delta Q\rangle|>0.5$ e/B. The highest coordinations number of six that may be achieved in a network of triangles reflects to some degree the vain attempt of neutral boron atoms to satisfy the octet rule. Ex- cess negative charge, with the maximum value $|\langle\Delta Q\rangle|=1.0 \mathrm{e} / \mathrm{B}$ considered here, offers the ability to satisfy this rule in the honeycomb network of $\epsilon_{6}$-B in Fig. 2(f).

To understand the chemical origin of these structural changes, we need to inspect the charge redistribution caused by additional doping, corresponding to the Fukui function for a crystal. Corresponding results for $\Delta \rho$ in selected electron doped borophene structures are presented in the SI. Complementing our results presented in Fig. 3 for borophene interacting with $\mathrm{Ca}_{2} \mathrm{~N}$, these results suggest that additional electrons are first accommodated in $p_{z}$ (or $\pi$ ) states normal to the borophene layer, which cause buckling, and then in $\sigma$ states, which reduce the amount of buckling. Starting with the lightly electron doped $\epsilon_{1}$-B containing triangles, we observe an increasing degree of buckling with increasing electron doping up 
to $|\langle\Delta Q\rangle| \lesssim 0.5 \mathrm{e} / \mathrm{B}$. At that point, the structure changes to the honeycomb structure mimicking hole-doped graphene. For $|\langle\Delta Q\rangle|>0.5 \mathrm{e} / \mathrm{B}$, additional excess charge gets increasingly accommodated in $\sigma$ states, which are much closer to $E_{F}$ in borophene than in graphene, causing a reduction of buckling down to zero for $\langle\Delta Q\rangle=-1.0 \mathrm{e} / \mathrm{B}$.

Assuming that the $\mathrm{Ca}_{2} \mathrm{~N}$ electride can transfer up to one electron per formula unit to a borophene layer, we can expect the maximum average charge $\langle\Delta Q\rangle$ per boron atom in $\mathrm{B} / \mathrm{Ca}_{2} \mathrm{~N}$ bilayers to range from $-0.22 \mathrm{e}$ in $\epsilon_{3^{-}}$ $\mathrm{B} / \mathrm{Ca}_{2} \mathrm{~N}$ to -0.28 e/atom in $\epsilon_{6}-\mathrm{B} / \mathrm{Ca}_{2} \mathrm{~N}$. The transferred charge may be up to twice as large in $\mathrm{Ca}_{2} \mathrm{~N} / \mathrm{B} / \mathrm{Ca}_{2} \mathrm{~N}$ sandwich structures. These values are slightly larger, but close to those found in the actual heterostructures, reported in Fig. 3. We compared the charge transfer between monolayers and multilayers of the $\mathrm{Ca}_{2} \mathrm{~N}$ electride in contact with borophene and found essentially no difference, as seen in the SI. Thus, the number of $\mathrm{Ca}_{2} \mathrm{~N}$ layers does not affect the maximum value of $\langle\Delta Q\rangle$.

It is not easy to achieve the doping level $\langle\Delta Q\rangle=-1.0$ e/B by contacting an electronegative material. According to our results for the $\mathrm{Ca}_{2} \mathrm{~N} / \mathrm{B} / \mathrm{Ca}_{2} \mathrm{~N}$ sandwich structure in Fig. 4(d), the $\mathrm{Ca}_{2} \mathrm{~N}$ electride can provide only up to $\approx 0.4$ electrons per boron atom, much less than the desired doping level of $1 \mathrm{e} / \mathrm{B}$. An alternative to $\mathrm{Ca}_{2} \mathrm{~N}$ is $\mathrm{Y}_{2} \mathrm{C}$, which can supply twice as many electrons as $\mathrm{Ca}_{2} \mathrm{~N}$, but is hard to exfoliate. Assuming maximum charge transfer from $\mathrm{Y}_{2} \mathrm{C}$ to borophene, $\mathrm{B}$ could receive up to 0.8 electrons in the $\mathrm{Y}_{2} \mathrm{C} / \mathrm{B} / \mathrm{Y}_{2} \mathrm{C}$ heterostructure. Such a large electron transfer should further augment the Coulomb attraction between borophene and $\mathrm{Y}_{2} \mathrm{C}$, thus further deducing the interlayer distance, as seen in Figs. 3(a) and 3(b), and increase the amount of electron transfer, possibly up to $1.0 \mathrm{e} / \mathrm{B}$.

We have mentioned electronic structure parallels between the honeycomb structure of $\epsilon_{6^{-}}$ $B$ carrying one extra electron per atom and graphene. Even though the system of $\pi$ electrons near $E_{F}$ and the Dirac cone in the corner of the Brillouin zone occur in both systems, there are notable differences between the sys- tems. In graphene, the top of the $\sigma$-band lies more than $3 \mathrm{eV}$ below $E_{F}$, whereas this energy difference is only $0.2 \mathrm{eV}$ in $\epsilon_{6}$-B. Apparently, the lower core charge of elemental boron is the main reason, why the $\sigma$ and $\pi$ bands are energetically closer than in graphene. As mentioned before, this results in an increased role of $\sigma$ states in electron doped borophene structures.

According to our results in Fig. 5(a), changing the doping level $\langle\Delta Q\rangle$ from -0.25 e to $+0.25 \mathrm{e}$ results in a $23 \%$ increase of the borophene lattice constant $a_{1}$. Conversely, we may speculate that changing the lattice constant should modulate the electron transfer from the electride to the borophene layer, thus changing the dipole moment normal to the interface. In that case, an in-plane vibration of the heterostructure will cause this dipole to oscillate and to emit an electromagnetic signal of the same frequency.

Clearly, the most drastic effect of electron doping is seen in the hexagonal borophene structure stabilized in $\mathrm{MgB}_{2}$, which is the cause of superconductivity in this system. Electron doping may also be used as a way to change structures in a predictable way. As an illustration, we find the $\chi_{3}$-B structure, ${ }^{8}$ which has been synthesized at $680 \mathrm{~K}$ on $\mathrm{Ag}(111)$, to be very similar to the electron-doped, buckled $\epsilon_{3}$-B structure. In that case, a simpler way to fabricate $\chi_{3}-\mathrm{B}$ may consist of bringing borophene in contact with an electride, allowing it to relax to $\epsilon_{3}-\mathrm{B}$ while electron doped. The final flat structure of $\chi_{3}$-B may evolve after the electride has been removed. These two examples illustrate new possibilities of using doping to change the structure of boron. Even though doping may have a lesser effect on the equilibrium structure of other systems, our findings about the interplay between structure and excess charge have a general validity.

\section{Summary and Conclusions}

In summary, we have studied the effect of electron doping on the bonding character and stability of two-dimensional (2D) structures of elemental boron, called borophene, which is known to form many stable allotropes. Our 
$a b$ initio calculations for the neutral system have revealed previously unknown stable $2 \mathrm{D} \epsilon$ $\mathrm{B}$ and $\omega$-B structures in addition to previously reported triangular lattices with monatomic vacancies. We found that the chemical bonding characteristic in this and other boron structures is strongly affected by extra charge, which is first accommodated in the $\pi$ and subsequently in the $\sigma$ network of electrons. Beyond a critical degree of electron doping, the most stable allotrope was found to change from $\epsilon$-B containing triangles and higher polygons to a buckled honeycomb structure. Additional electron doping, mimicking a transformation of boron to carbon, causes a gradual decrease in the degree of buckling of the honeycomb lattice that can be interpreted as piezoelectric response. We also found that net electron doping can be achieved by placing borophene in direct contact with layered electrides such as $\mathrm{Ca}_{2} \mathrm{~N}$. We found that electron doping can be doubled to $\approx 0.4 \mathrm{e} / \mathrm{B}$ atom by changing from the $\mathrm{B} / \mathrm{Ca}_{2} \mathrm{~N}$ bilayer to the $\mathrm{Ca}_{2} \mathrm{~N} / \mathrm{B} / \mathrm{Ca}_{2} \mathrm{~N}$ sandwich geometry.

\section{Computational Techniques}

Our calculations of the stability, equilibrium structure and electronic structure have been performed using density functional theory (DFT) as implemented in the SIESTA ${ }^{30}$ code. Periodic boundary conditions have been used throughout the study, with monolayers represented by a periodic array of slabs separated by a $30 \AA$ thick vacuum region. We used the Perdew-Burke-Ernzerhof (PBE) $)^{31}$ exchangecorrelation functional. The SIESTA calculations used norm-conserving Troullier-Martins pseudopotentials, $\stackrel{32}{,}$ a double- $\zeta$ basis including polarization orbitals, and a mesh cutoff energy of 250 Ry to determine the self-consistent charge density, which provided us with a precision in total energy of $\lesssim 2 \mathrm{meV} /$ atom. The reciprocal space has been sampled by a fine $\operatorname{grid}^{33}$ of $4 \times 4 k$-points in the $2 \mathrm{D}$ Brillouin zones (BZ) of the primitive unit cells of neutral and doped borophene containing 32 atoms, $3 \times 3 k$ points in the BZ of supercell of heterostructure of $\mathrm{Ca}_{2} N$ and $\epsilon_{6}$, and $3 \times 1 k$-points in the BZ of supercell of heterostructure of $\mathrm{Ca}_{2} \mathrm{~N}$ and $\epsilon_{3}$. Geometries have been optimized using the conjugate gradient (CG) method, $\stackrel{34}{ }$ until none of the residual Hellmann-Feynman forces exceeded $10^{-2} \mathrm{eV} / \AA$.

\section{Supporting Information Avail- able}

The following files are available free of charge. Additional results are presented for the characterization of borophene layers under conditions not covered in the main manuscript. These include optimum geometries and charge distribution in free-standing electron doped borophene as well as in vertical heterostructures of borophene and $\mathrm{Ca}_{2} \mathrm{~N}$ bilayers. Also presented are electronic band structures of systems described in the main manuscript, results characterizing the effect of strain on the electronic band structure, and charge density differences corresponding to the Fukui function for selected doped borophene allotropes.

\section{Author Information}

\section{Corresponding Author}

${ }^{*}$ E-mail: tomanek@pa.msu.edu

\section{Notes}

The authors declare no competing financial interest.

Acknowledgement The authors acknowledge financial support by the NSF/AFOSR EFRI 2-DARE grant number EFMA-1433459. We thank Xianqing Lin, Andrii Kyrylchuk and Rick Becker for many enriching discussions. Computational resources have been provided by the Michigan State University High Performance Computing Center.

\section{References}

(1) Dresselhaus, M. S.; Dresselhaus, G. Adv. Phys. 1981, 30, 139-326.

(2) Py, M. A.; Haering, R. R. Can. J. Phys. 1983, 61, 76-84. 
(3) Wu, X.; Dai, J.; Zhao, Y.; Zhuo, Z.; Yang, J.; Zeng, X. C. ACS Nano 2012, $6,7443-7453$.

(4) Quandt, A.; Boustani, I. ChemPhysChem. 2005, 6, 2001-2008.

(5) Evans, M. H.; Joannopoulos, J. D.; Pantelides, S. T. Phys. Rev. B 2005, 72, 045434.

(6) Kunstmann, J.; Quandt, A. Phys. Rev. B 2006, 74, 035413.

(7) Mannix, A. J.; Zhou, X.-F.; Kiraly, B.; Wood, J. D.; Alducin, D.; Myers, B. D.; Liu, X.; Fisher, B. L.; Santiago, U.; Guest, J. R.; Yacaman, M. J.; Ponce, A.; Oganov, A. R.; Hersam, M. C.; Guisinger, N. P. Science 2015, 350, 1513-1516.

(8) Feng, B.; Zhang, J.; Zhong, Q.; Li, W.; Li, S.; Li, H.; Cheng, P.; Meng, S.; Chen, L.; Wu, K. Nat. Chem. 2016, 8, 563-568.

(9) Zhang, Z.; Penev, E. S.; Yakobson, B. I. Chem. Soc. Rev. 2017, 46, 6746-6763.

(10) Zhou, X.-F.; Dong, X.; Oganov, A. R.; Zhu, Q.; Tian, Y.; Wang, H.-T. Phys. Rev. Lett. 2014, 112, 085502.

(11) Zhou, X.-F.; Oganov, A. R.; Shao, X.; Zhu, Q.; Wang, H.-T. Phys. Rev. Lett. 2014, 113, 176101.

(12) Ma, F.; Jiao, Y.; Gao, G.; Gu, Y.; Bilic, A.; Chen, Z.; Du, A. Nano Lett. 2016, 16, 3022-3028.

(13) Li, W.; Kong, L.; Chen, C.; Gou, J.; Sheng, S.; Zhang, W.; Li, H.; Chen, L.; Cheng, P.; Wu, K. Sci. Bull. 2018, 63, 282-286.

(14) Nishibori, E.; Takata, M.; Sakata, M.; Tanaka, H.; Muranaka, T.; Akimitsu, J. J. Phys. Soc. Japan 2001, 70, 2252-2254.
(15) Kappera, R.; Voiry, D.; Yalcin, S. E.; Branch, B.; Gupta, G.; Mohite, A. D.; Chhowalla, M. Nat. Mater. 2014, 13, 1128.

(16) Perkins, P. G. In Boron and Refractory Borides; Matkovich, V., Ed.; Springer: Berlin, 1977; Chapter 3, pp 31-51.

(17) Kolmogorov, A. N.; Curtarolo, S. Phys. Rev. B 2006, 74, 224507.

(18) Nagamatsu, J.; Nakagawa, N.; Muranaka, T.; Zenitani, Y.; Akimitsu, J. $\mathrm{Na}$ ture 2001, 410, 63-64.

(19) Tang, H.; Ismail-Beigi, S. Phys. Rev. Lett. 2007, 99, 115501.

(20) Tang, H.; Ismail-Beigi, S. Phys. Rev. B 2009, 80, 134113.

(21) Tang, H.; Ismail-Beigi, S. Phys. Rev. B 2010, 82, 115412.

(22) Karmodak, N.; Jemmis, E. D. Angew. Chem. Int. Ed. 2017, 56, 10093-10097.

(23) Tarkowski, T.; Majewski, J. A.; Szwacki, N. G. FlatChem 2018, \%, $42-47$.

(24) Tan, X.; Tahini, H. A.; Smith, S. C. ACS Appl. Mater. Interfaces 2017, 9, 1982519830.

(25) Druffel, D. L.; Kuntz, K. L.; Woomer, A. H.; Alcorn, F. M.; Hu, J.; Donley, C. L.; Warren, S. C. J. Am. Chem. Soc. 2016, 138, 16089-16094.

(26) Lee, K.; Kim, S. W.; Toda, Y.; Matsuishi, S.; Hosono, H. Nature 2013, 494, 336-340.

(27) Zhang, X.; Xiao, Z.; Lei, H.; Toda, Y.; Matsuishi, S.; Kamiya, T.; Ueda, S.; Hosono, H. Chem. Mater. 2014, 26, 66386643 .

(28) Nixon, D. E.; Parry, G. S. J. Phys. C: Solid State Phys. 1969, 2, 1732-1742. 
(29) Liu, D.; Every, A. G.; Tománek, D. Phys. Rev. B 2016, 94, 165432.

(30) Artacho, E.; Anglada, E.; Dieguez, O.; Gale, J. D.; Garcia, A.; Junquera, J.; Martin, R. M.; Ordejon, P.; Pruneda, J. M.; Sanchez-Portal, D.; Soler, J. M. J. Phys. Cond. Mat. 2008, 20, 064208.

(31) Perdew, J. P.; Burke, K.; Ernzerhof, M. Phys. Rev. Lett. 1996, 77, 3865-3868.

(32) Troullier, N.; Martins, J. L. Phys. Rev. B 1991, 43, 1993-2006.

(33) Monkhorst, H. J.; Pack, J. D. Phys. Rev. $B$ 1976, 13, 5188-5192.

(34) Hestenes, M. R.; Stiefel, E. J. Res. Natl. Bur. Stand. 1952, 49, 409-436. 